\title{
Influence of Skin Diseases on Fingerprint Recognition
}

\author{
Martin Drahansky, ${ }^{1}$ Michal Dolezel, ${ }^{1}$ Jaroslav Urbanek, ${ }^{2}$ \\ Eva Brezinova, ${ }^{3}$ and Tai-hoon Kim $^{4}$ \\ ${ }^{1}$ Faculty of Information Technology, Brno University of Technology, Bozetechova 2, 61266 Brno, Czech Republic \\ ${ }^{2}$ Faculty of Medicine and Dentistry, Palacký University and Faculty Hospital, I. P. Pavlova 6, 77520 Olomouc, Czech Republic \\ ${ }^{3}$ Faculty of Medicine, Masaryk University, Komenského náměstí 2, 66243 Brno, Czech Republic \\ ${ }^{4}$ Department of Multimedia Engineering, Hannam University, 133 Ojeong-dong, Daedeok-gu, Daejeon 306791, Republic of Korea \\ Correspondence should be addressed to Martin Drahansky, drahan@fit.vutbr.cz
}

Received 25 February 2012; Accepted 26 February 2012

Academic Editor: Sabah Mohammed

Copyright () 2012 Martin Drahansky et al. This is an open access article distributed under the Creative Commons Attribution License, which permits unrestricted use, distribution, and reproduction in any medium, provided the original work is properly cited.

\begin{abstract}
There are many people who suffer from some of the skin diseases. These diseases have a strong influence on the process of fingerprint recognition. People with fingerprint diseases are unable to use fingerprint scanners, which is discriminating for them, since they are not allowed to use their fingerprints for the authentication purposes. First in this paper the various diseases, which might influence functionality of the fingerprint-based systems, are introduced, mainly from the medical point of view. This overview is followed by some examples of diseased finger fingerprints, acquired both from dactyloscopic card and electronic sensors. At the end of this paper the proposed fingerprint image enhancement algorithm is described.
\end{abstract}

\section{Introduction}

Skin diseases represent very important but often neglected factor of the fingerprint acquirement. It is impossible to say in general how many people suffer from skin diseases, because there are so many various skin diseases [1-5]. In a general medical practice, about $20-25 \%$ of patients with skin complaints are referred. When discussing whether the fingerprint recognition technology is a perfect solution capable to resolve all our security problems, we should always keep in mind those potential users who suffer from some skin disease.

In the following text, several skin diseases, which attack hand palms and fingertips, are introduced from the medical point of view.

The situation after successful recovery of a potential user from such skin diseases is, however, very important for the possible further use of fingerprint recognition devices. If the disease has attacked and destroyed the structure of papillary lines in the epidermis and underlying dermis (top two layers of the skin), the papillary lines will not grow in the same form as before (if at all), and therefore such users could be restricted in their future life by being excluded from the use of fingerprint recognition systems, though their fingers do not have any symptoms of the skin disease anymore.

\section{Skin Diseases}

In the process of the fingerprint image acquirement, the skin structure on the fingertip is being scanned. Skin is remarkable organ of the body, which is able to perform various vital functions. It can mold to different shapes, stretch, and harden, but can also feel a delicate touch, pain, pressure, hot, and cold, and is an effective communicator between the outside environment and the brain.

Skin makes up to $12-15 \%$ of an adult's body weight. Each square centimeter has 6 million cells, 5,000 sensory points, 100 sweat glands, and 15 sebaceous glands. It consists of three layers (see Figure 1) [6]: epidermis (the outer layer), dermis ("true skin"), and subcutaneous (fat) layer.

Skin is constantly being regenerated [6]. A skin cell starts its life at the lower layer of the skin (the basal layer of the dermis), which is supplied with blood vessels and nerve endings. The cell migrates upward for about two weeks until it reaches the bottom portion of the epidermis, which is the 


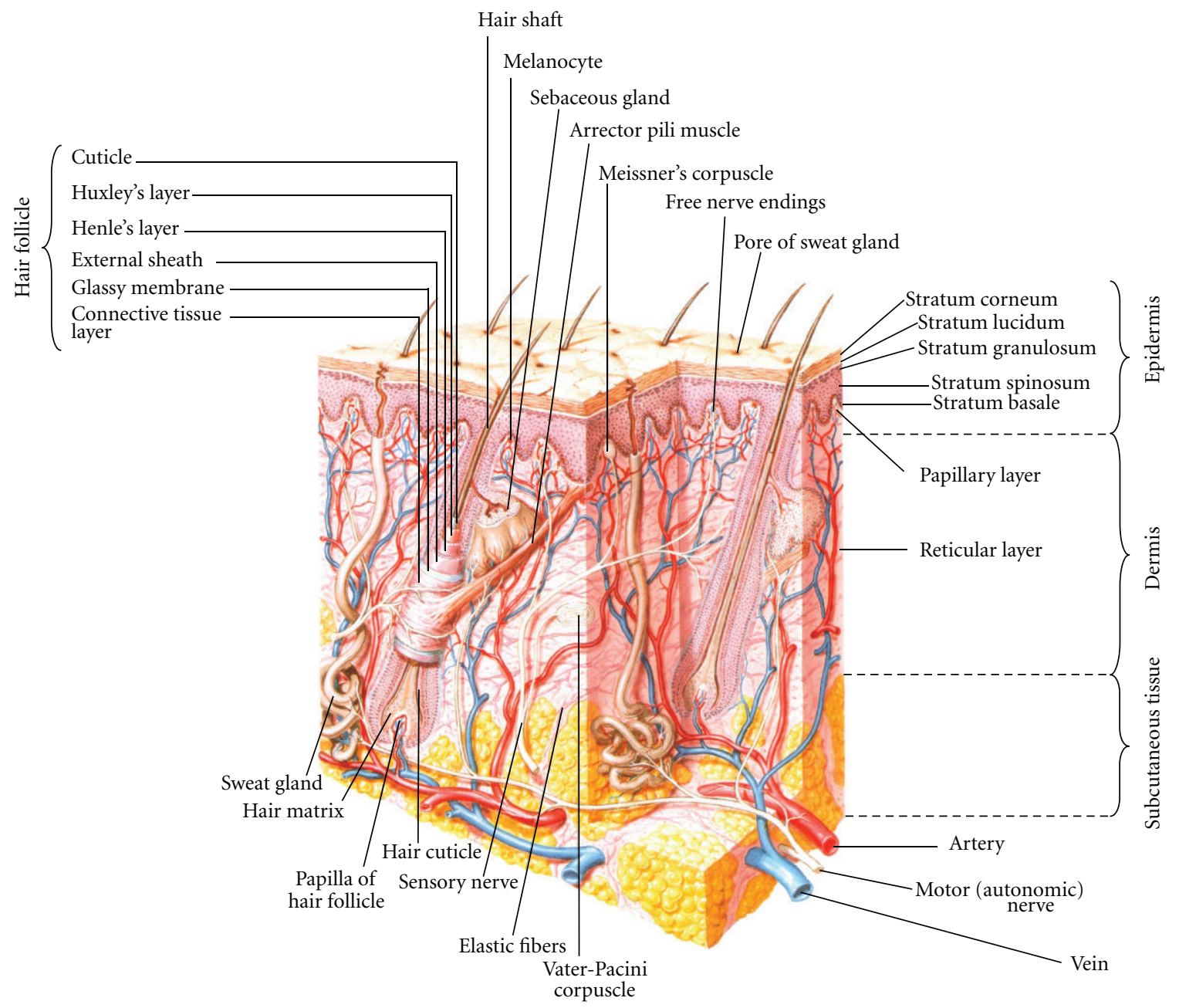

Figure 1: Skin structure [5].

outermost skin layer. The epidermis is not supplied with blood vessels but has nerve endings. For another 2 weeks, the cell undergoes a series of changes in the epidermis, gradually flattening out and moving toward the surface. Then it dies and is shed. There are six skin functions [6] as follows.

Sensation. The nerve endings in the skin detect touch, heat, cold, pain, and light pressure.

Heat Regulation. Skin helps to regulate the body temperature by sweating to cool the body down when it overheats and by shivering creating "goose bumps" when it is cold. Shivering closes the pores. The tiny hair that stands on end traps warm air and thus helps keep the body warm.

Absorption. Absorption of ultraviolet rays from the sun helps to form vitamin D in the body, which is vital for bone formation. Some creams, essential oils, and medicines (e.g., antismoking patches) can also be absorbed through the skin into the blood stream.
Protection. The skin protects the body from ultraviolet light - too much of it is harmful to the body — by producing a pigment called melanin. It also protects us from the invasion of bacteria and germs by forming an acid mantle (formed by the skin sebum and sweat). This barrier also prevents moisture loss.

Excretion. Waste products and toxins are eliminated from the body through the sweat glands. It is a very important function which helps to keep the body "clean" from the inside.

Secretion. Sebum and sweat are secreted onto the skin surface. The sebum keeps the skin lubricated and soft, and the sweat combines with the sebum to form an acid mantle which creates the right $\mathrm{pH}$ balance for the skin to fight off infection.

There are a lot of skin diseases, which can affect palms and fingers. These diseases can be divided into two groups. In the first group, palms and fingers are mainly affected. In the second group, skin lesions can be diffuse, involving wide 
areas. We can also differentiate skin diseases $[7,8]$ by type or localization of histopathological changes in epidermis and dermis. Some of these diseases together with photographs are described in the following chapters. These clearly show that skin diseases may cause many problems in automatic biometric systems.

\subsection{Diseases Causing Histopathological Changes of Epidermis} and Dermis. These diseases may cause problems for the most types of sensors, because color of the skin and structure of epidermis and dermis are influenced.

Hand eczema [5, 9-11] is an inflammatory noncontagious chronic disease with relapsing course. It is one of the most common problems encountered by the dermatologist. Pathological changes in the epidermis and in upper dermis produce distinctive clinical pictures. Acute form is characterized by presence of erythema, swelling, blisters, and crusts. In chronic form, scaling, fissuring, and lichenification may be seen. We can find several main forms of hand eczema: irritant dermatitis (acute or chronic), allergic dermatitis (acute or chronic), atopic eczema and hyperkeratotic dermatitis. Hand dermatitis causes discomfort and embarrassment and, because of its locations, interferes significantly with normal daily activities. Hand dermatitis is common in many industrial occupations. Typical example is hand dermatitis in construction and cement workers.

The prevalence of hand eczema is approximately $5.4 \%$. The most common type of hand eczema is irritant contact dermatitis (35\%; see Figure 2), followed by atopic eczema (22\%) and allergic contact dermatitis (19\%). The most common contact allergies are to nickel, cobalt, fragrance mix, balsam of Peru, epoxy resin, and colophony. Hand eczema was more common among people reporting occupational exposure. Housewives, hairdressers, nurses, workers with repeated exposure to cement, mortar, cutting oils, or abrasive are mainly involved. Hand eczema represents a major occupational problem and accounts for more than $80 \%$ of all occupational dermatitis. Patients with hand eczema frequently miss work and may need to change occupations.

Fingertip eczema [5] is very dry, chronic form of eczema of the palmar surface of the fingertips. It may be result of an allergic reaction or may occur in children and adults as an isolated phenomenon of unknown cause. One finger or several fingers may be involved. Initially the skin may be moist and then become dry, cracked, and scaly. The skin peels from the fingertips distally, exposing a very dry, red, cracked, fissured, tender, or painful surface without skin lines.

Pompholyx (dishidrosis) [4] is a distinctive idiopathic reaction pattern presenting as usually symmetric vesicular hand and foot dermatitis. It is one of the most common skin disorders. It is not related to blockage of sweat ducts, although palmoplantar hyperhidrosis is common in these patients. Itching precedes the appearance of tiny water-filled vesicles on the palms and sides of the fingers which are relatively deep seated. The skin may be red and wet (see Figure 3 ). The vesicles slowly resolve and are replaced by erythematous scaly patches. Chronic eczematous changes with erythema, scaling, and lichenification may follow.

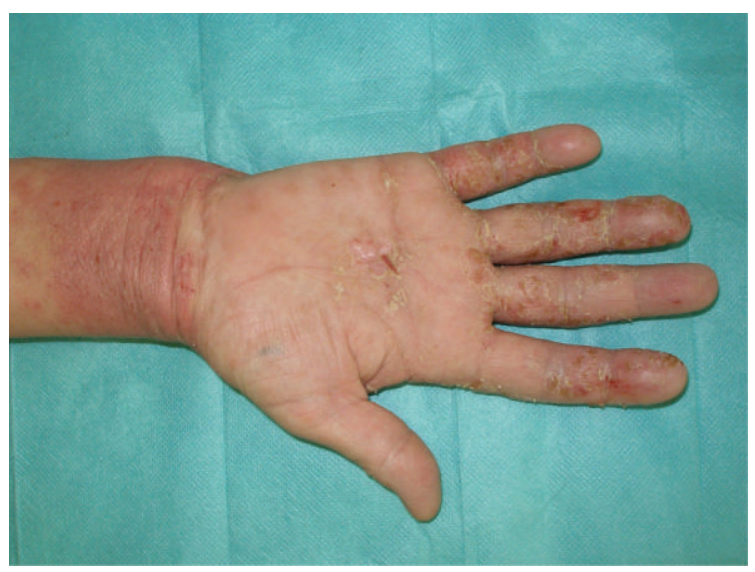

Figure 2: Hand eczema.

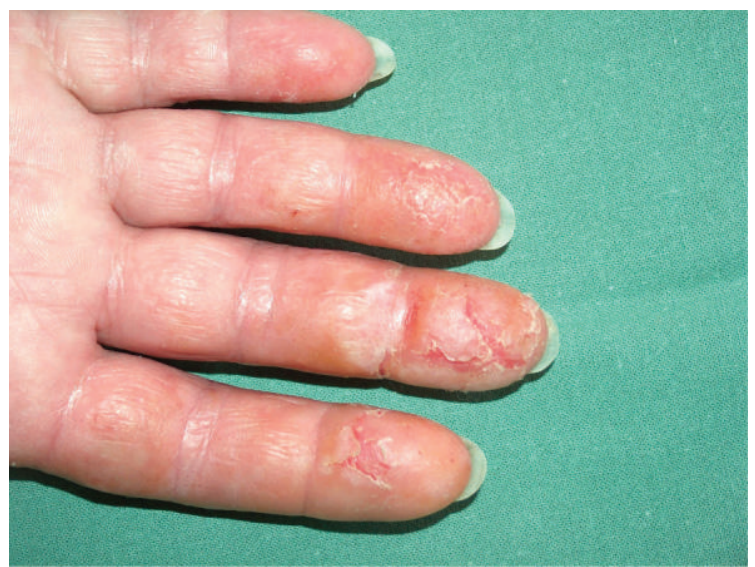

Figure 3: Pompholyx (dishidrosis).

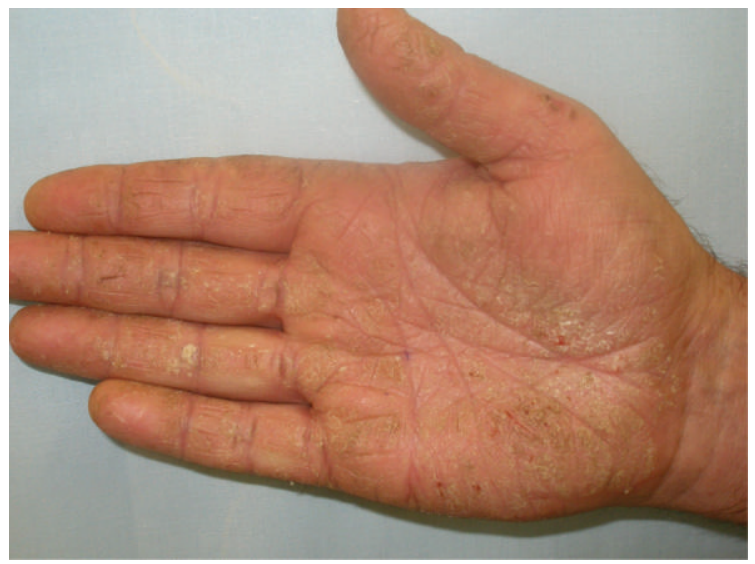

FIGURE 4: Tinea of the palm.

Etiology is often unclear. There are some possible causes like atopic diathesis, tinea pedis, contact allergy, or systemic allergic reaction.

Tinea of the palm (tinea manus) $[5,10,11]$. The most common variant is dry keratotic form of tinea characterized by adherent scales on an erythematous background. The nail involvement is usually present. The skin changes are often 


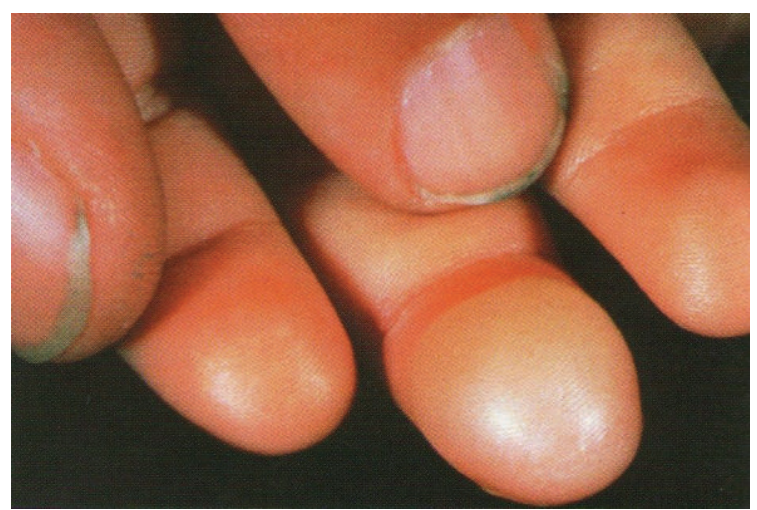

Figure 5: Pyoderma [4, page 262].

unilateral, involving only one hand. The affected hand is usually the dominant one (figure 4).

The dry keratotic form may be asymptomatic, and the patient may be unaware of the infection, attributing the dry, thick, scaly surface to hard physical labor. It is frequently seen in association with tinea pedis whose prevalence is 10 to $30 \%$. Erythema and scaling of the right hand can be seen in Figure 3.

Pyoderma $[9,10]$ is a sign of bacterial infection of the skin. It is caused by Staphylococcus aureus or Streptococcus pyogenes. Blistering distal dactylitis is specific type of pyoder$\mathrm{ma}$ and is characterized by tense superficial blisters occurring on a tender erythematous base over the volar fat pad of the phalanx of a finger (see Figure 5).

It is more common for children, and some people are more susceptible to these diseases (such as diabetics, alcoholics, HIV patients, etc.). Typically the face and hands are affected. The lesions have red color with blisters or filled by pus. Felon is soft tissue infection of pulp space of distal phalanx. In Figure 6 you can see cellulites on the fingertip, which arose following acute paronychia of lateral nail fold and can involve the whole distal phalanx.

Pitted keratolysis [5] is a disease mimicking tinea, especially for people who swelter and wear rubber gloves in the hot, humid environment. Hyperhidrosis is the most frequently observed symptom. The disease is bacterial in origin (Corynebacterium species), characterized by many circular or longitudinal and punched out depressions in the skin surface. The eruption is limited to the stratum corneum (see Figure 7).

Keratolysis exfoliativa [5] is a common, chronic, asymptomatic, noninflammatory, bilateral peeling of the palms of the hands. Its cause is unknown. The eruption is most common during the summer months and is often associated with sweaty palms and soles. It is characterized by scaling and peeling; the central area becomes slightly red and tender.

Lichen planus [10] is quite common, unique inflammatory cutaneous and mucous membrane reaction pattern of unknown etiology. The prevalence of this skin disease is around $0.5 \%$. Lichen planus of the palm and soles generally occurs as an isolated phenomenon. The lesions are papules aggregated into semitranslucent hyperkeratotic or verrucous plaques with globular waxy surface; ulceration may occur.

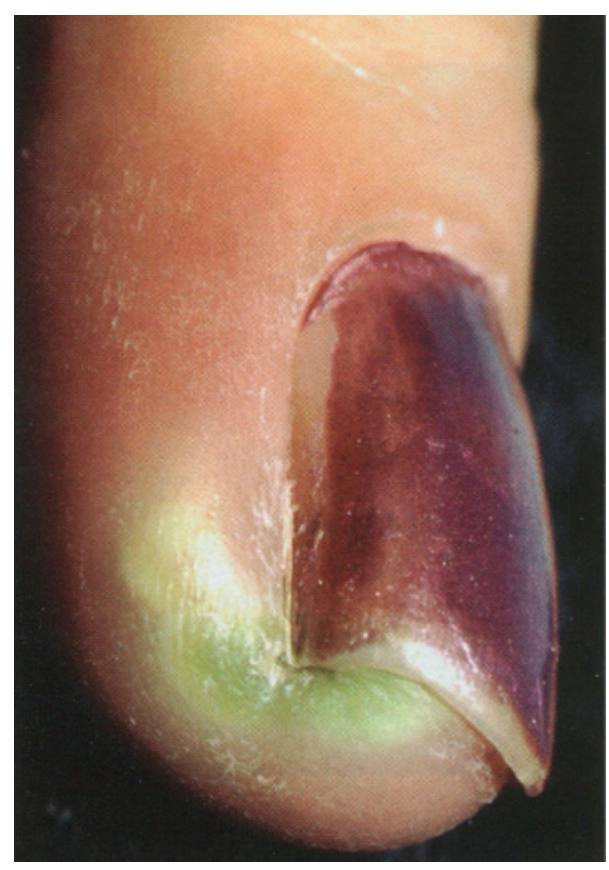

FIGURE 6: Cellulite of the fingertip [9, page 1000].

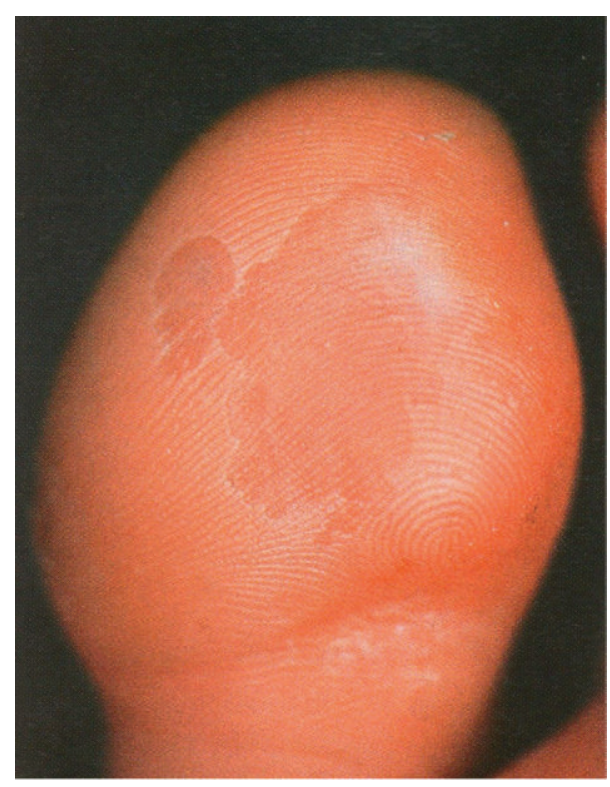

Figure 7: Pitted keratolysis [4, page 269].

The lesions are often painful. This form of lichen planus is very therapy resistant.

Acanthosis nigricans $[4,9]$ is nonspecific uncommon reaction pattern that may accompany obesity, diabetes, and tumors. Acanthosis nigricans is classified into benign and malignant forms. In all cases the disease presents with symmetric, brown thickening of the skin (see Figure 8). During the process, there is papillary hypertrophy, hyperkeratosis, and increased number of melanocytes in the epidermis. The palmar ridges of the palm show maximal accentuation, thus 


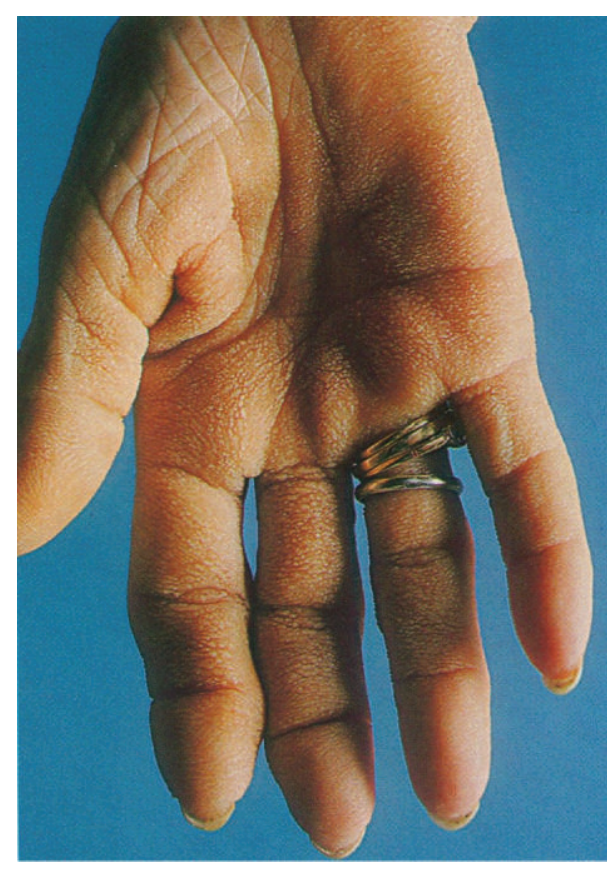

Figure 8: Acanthosis nigricans [9, page 502].

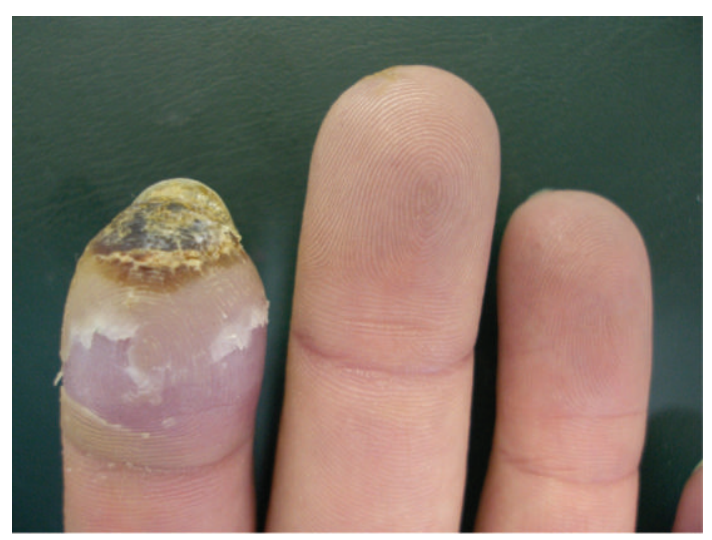

Figure 9: Systemic sclerosis.

resembling the mucosa of the stomach of a ruminant (tripe palm).

Pyogenic granuloma [5] is a benign acquired vascular lesion of the skin that is common in children and young adults. It often appears as a response to an injury or hormonal factors. Lesions are small rapidly growing, yellow-to-bright red, dome shaped. The fingers and palms are very often involved. The lesions can be bleeding or have the crust on the surface.

Systemic sclerosis $[9,10]$ is a chronic autoimmune disease characterized by sclerosis of the skin or other organs. Emergence of acrosclerosis is decisive for fingerprinting. Initially the skin is infused with edema mainly affecting hands. With the progressive edema stiff skin appears and necrosis of fingers may form (see Figure 9).

The disease leads to sclerodactyly with contractures of the fingers. Typical "rat bite" necroses and ulcerations of fingertips may develop, healing with pitted scars. Cutaneous

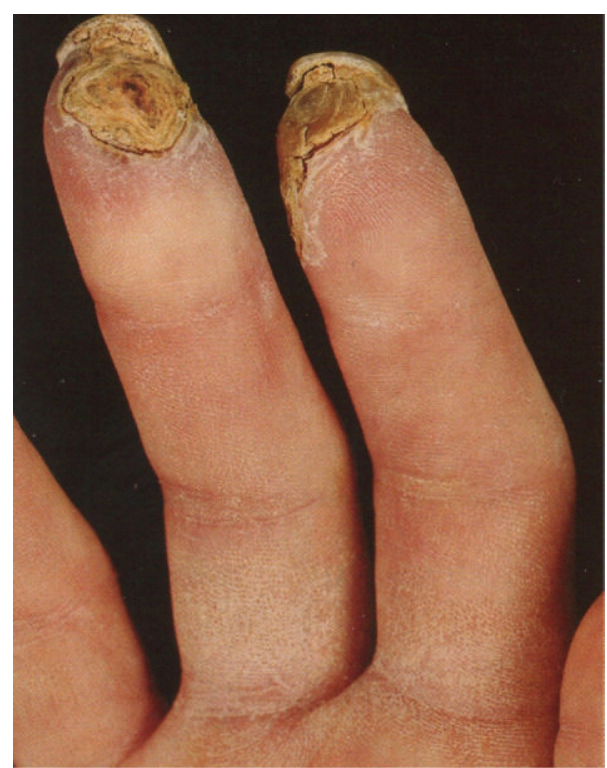

Figure 10: Raynaud's phenomenon [9, page 405].

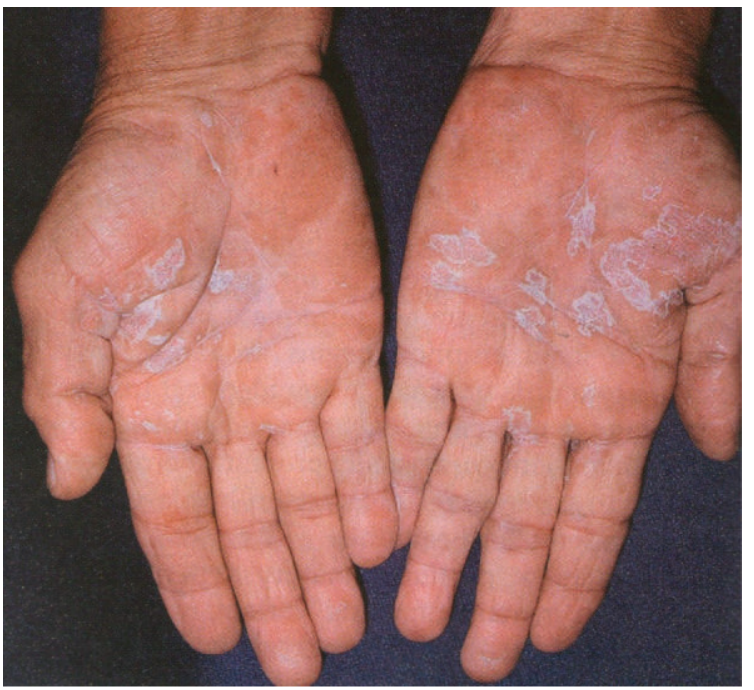

FIgURE 11: Drug-included skin reaction to gold [4, page 128].

calcification occurs on fingertips and may ulcerate and exude white paste. Involvement of fingers is usually seen in early stage of limited systemic scleroderma. For more than $90 \%$ of patients is typical Raynaud's phenomenon (see Figure 10). The typical patient is a $50+$ years old woman.

Raynaud's phenomenon $[5,9,10]$ represents an episodic vasoconstriction of the digital arteries and arterioles that is precipitated by cold and stress. Raynaud's phenomenon can be associated with other disease, above all connective tissue disease. Persistent vasospasm of medium-sized arterioles can sometimes lead to gangrene of the terminal digits as illustrated in Figure 10.

It is much more common in women. There are three stages during a single episode: pallor (white), cyanosis (blue), 


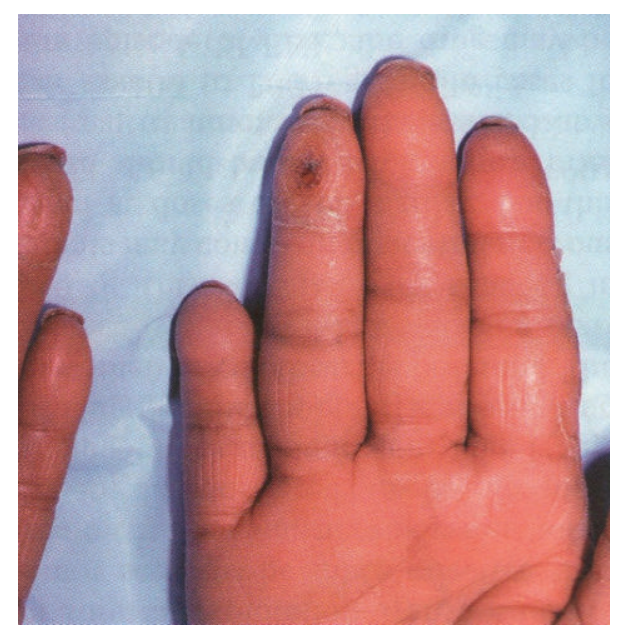

FIgURE 12: Leprosy-loss of tissue [4, page 351].

and hyperemia (red). Estimates of the prevalence of Raynaud's phenomenon ranged between 5 and $20 \%$. The therapy of this disorder is not quite satisfactory and problems are usually long lasting. There are a lot of causes of Raynaud's phenomenon-connective tissue diseases, obstructive arterial diseases, neurogenic disorders, drugs as beta-adrenergic blockers and ergot preparations, cytostatics, trauma, hematologic causes, and so forth.

Drug-induced skin reactions [5] are among the most common adverse drug reactions. They occur in many forms and can mimic virtually any dermatosis and occur in $2-3 \%$ of hospitalized patients. Sulfonamides, NSAIDs, and anticonvulsants are most often applied in the etiology. Figure 11 shows lichenoid drug reaction to gold.

Leprosy [9] is a chronic granulomatous disease caused by $M$. leprae, principally acquired during childhood/young adulthood. Clinical manifestations, natural history, and prognosis of leprosy are related to the host response, and various types of leprosy represent the spectra of the host's immunologic response. It is disease of developing world, 600,000 new cases annually and 1.5 to 8 million total cases worldwide. More than $80 \%$ of cases occur in India, China, Myanmar, Indonesia, Brazil, and Nigeria. Lepromatous type can lead to loss of tissue of fingertips (see Figure 12).

Herpes simplex virus $[4,9]$ in the host with systemic immune-compromise may cause chronic ulcerations as you can see by the patient with advanced HIV disease in Figure 13(a).

Herpetic infection may uncommonly occur on the fingers or periungually. Lesions begin with tenderness, and erythema- and deep-seated blisters develop 24 to $48 \mathrm{~h}$ after symptoms begin (see Figure 13(b)).

Scabies $[10,12]$ is highly contagious disease caused by the mite Sarcoptes scabiei. It is characterized by red papules, vesicles, and crusts located usually on the areas with tender skin, palms, and soles especially in infants.

Erythema multiforme $[10,11]$ is quite common skin disorder with multifactorial cause (see Figure 14). The most common triggering agents are infections (in the first place herpes virus) and drugs. Minor and major variant of this

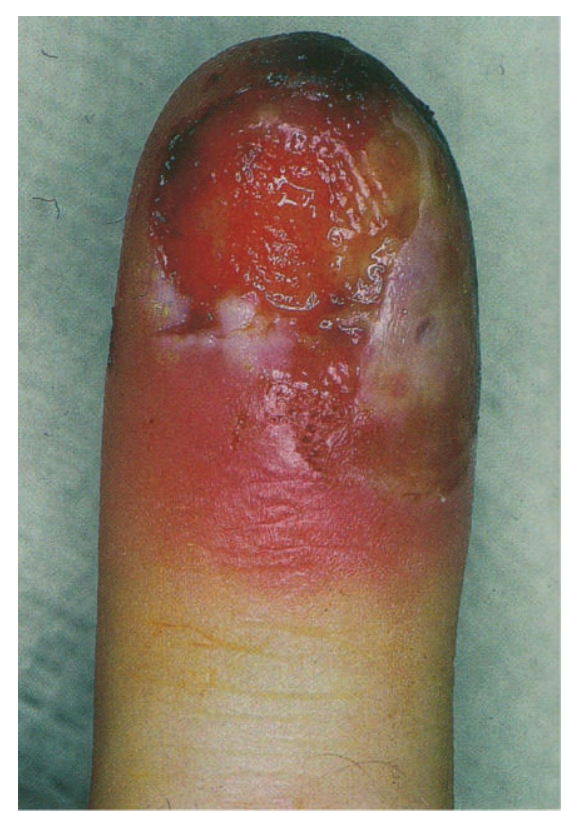

(a)

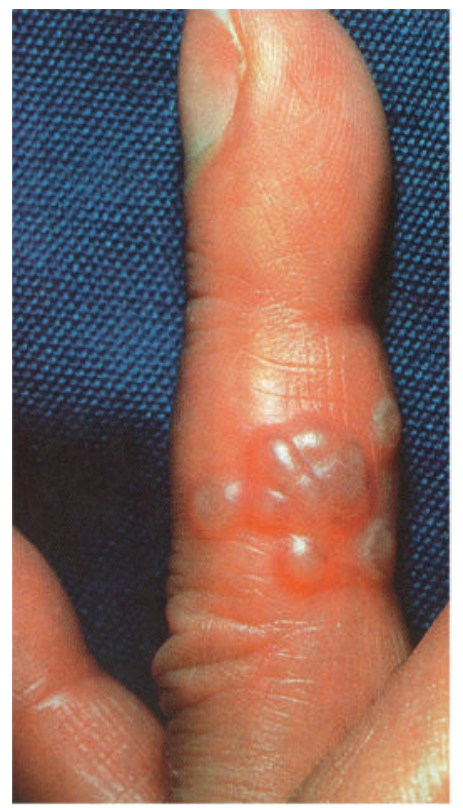

(b)

Figure 13: Herpes simplex virus. (a) patient with HIV [9, page 813]; (b) deep-seated blisters [4, page 369].

disease is described. Both forms are characterized by erythematous target-shaped lesions with a center with hemorrhage, blistering, necrosis, or crust. When the trigger is herpetic infection, frequent recurrences come.

Dermatitis artefacta [11] are changes of skin due to the manipulation by patient. Patients often have psychosomatic, psychiatric, or drug-abuse problems.

2.2. Diseases Causing Skin Discoloration. These diseases are focused mainly on optical sensors. 


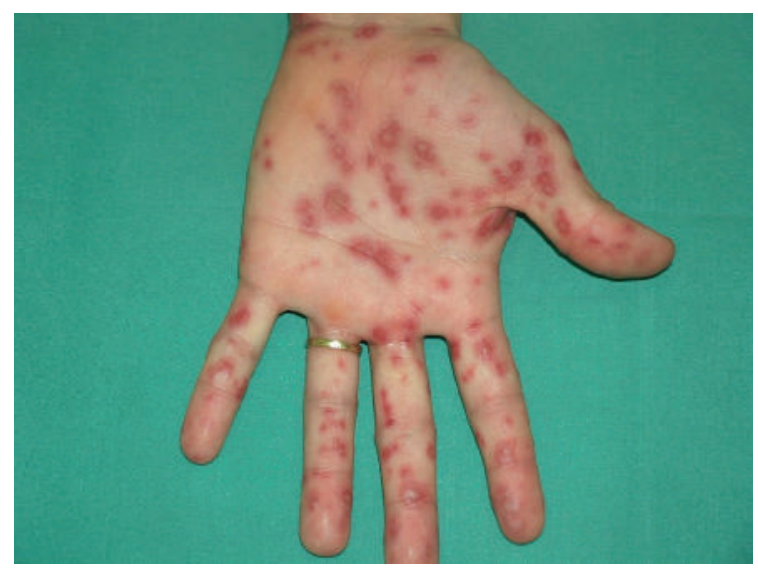

FIGURE 14: Erythema multiforme.

Hand, foot, and mouth disease (HFMD) $[4,5]$ is a contagious enteroviral infection occurring primarily in children and characterized by a vesicular palmoplantar eruption. The skin lesions begin as red macules that rapidly become pale, white, oval vesicles with red areola.

Xanthomas $[5,9,10]$ are lipid deposits in the skin and tendons that occur secondary to abnormalities of lipid metabolism. Xanthoma striatum palmare is pathognomonic for familial dysbetalipidemia, which is prone to atherosclerotic cardiovascular disease. Palmar xanthomas can also follow multiple myeloma and biliary cirrhosis. These localized deposits are yellow-orange, flat or elevated, and are frequently very firm, localized on the volar creases of palms and fingers.

Carotenosis [4] is yellowish discoloration of the skin, especially of the palms and soles that is sometimes seen in diabetic patients.

Scarlet fever (scarlatina) $[5,10]$ is contagious worldwide disease caused by $\beta$-hemolytic Streptococcus that produces an erythrogenic toxin. It is most common in children (ages 1 to 10 years). In the ending stages of the disease, large sheets of epidermis may be shed from the palms in glove-like cast, exposing new tender and red epidermis beneath.

Kawasaki's disease [9] is an acute febrile illness of infants and children, characterized by cutaneous and mucosal erythema and edema with subsequent desquamation and cervical lymphadenitis and complicated by coronary artery aneurysms (20\%). Most cases of Kawasaki's disease in adults represent toxic shock syndrome. Erythematous macules appear 1 to 3 days after onset of fever, enlarge, and become more numerous; then desquamation beginning on tips of fingers is highly characteristic (see Figure 15).

Secondary syphilis $[9,10]$ (see Figure 16) starts at about the 9th week of infection and is characterized by mucocutaneous lesions, which may assume a variety of shapes, including round, elliptic, or annular. These lesions are called syphilids. Semirigid small lesions of red-brown color with scaling may be observed on palms, soles, and fingers. Papulosquamous eruption can be similar to psoriasis.

Hereditary hemorrhagic telangiectasia [9] is an autosomal dominant condition affecting blood vessels, especially in the mucous membranes of the mouth and the gastrointestinal

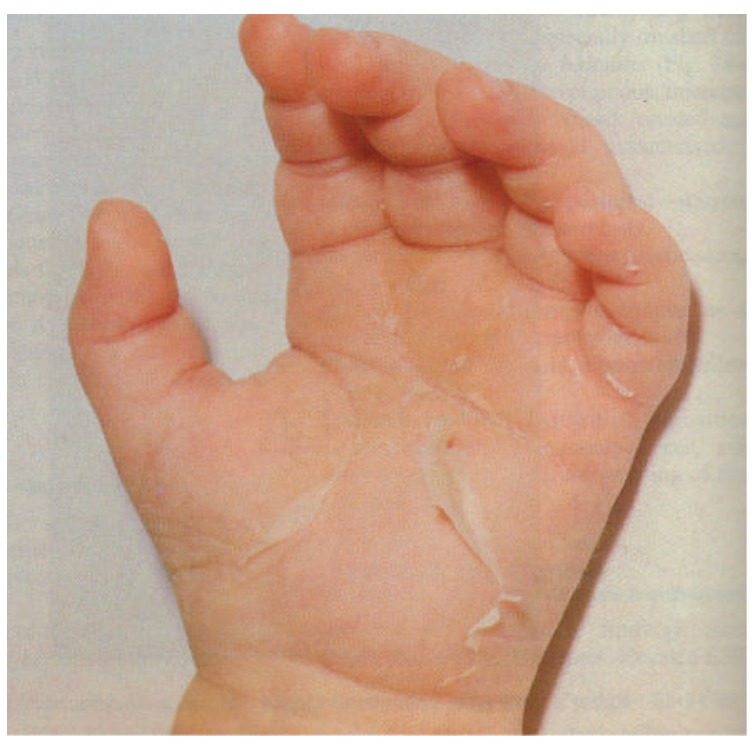

FIgURE 15: Kawasaki's disease [9, page 425].

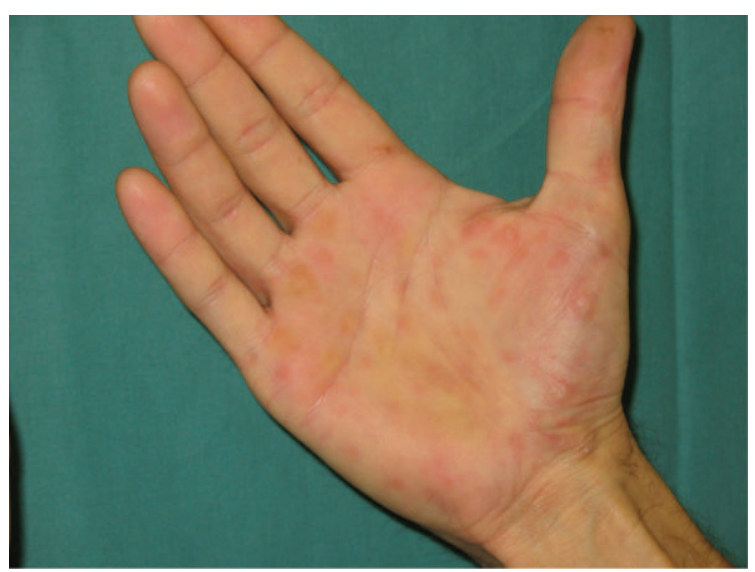

Figure 16: Secondary syphilis.

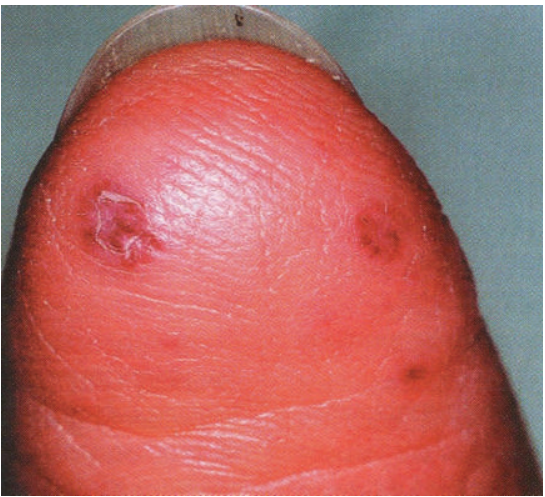

(a)

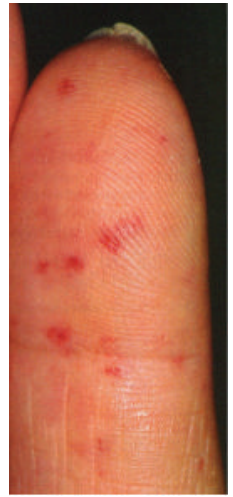

(b)
FIgURE 17: Hereditary hemorrhagic Telangiectasia ((a) [4, page 844], (b) [9, page 468]). 


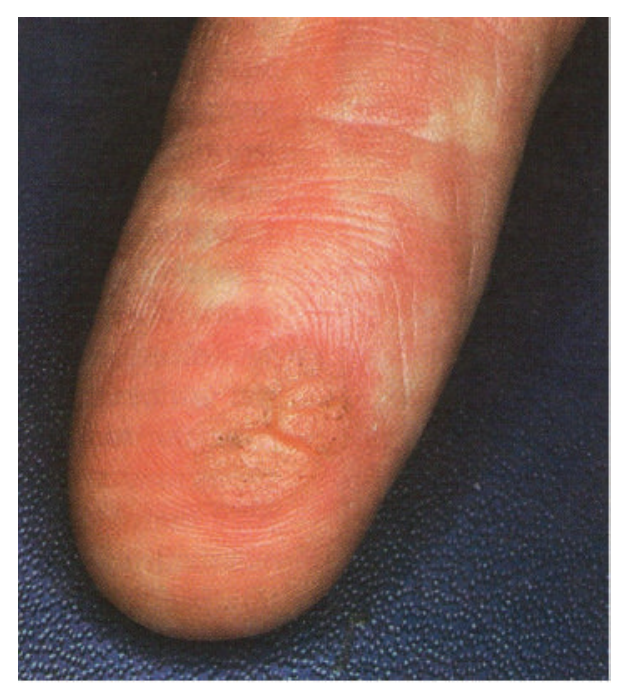

Figure 18: Warts [4, page 404].

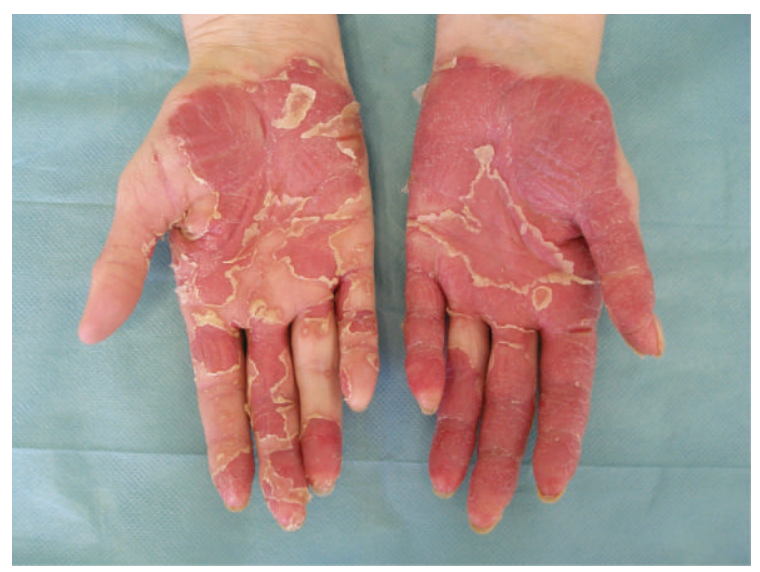

Figure 19: Psoriasis.

tract. The diagnostic lesions are small, pulsating, macular, and papular, usually punctuate. Telangiectases are present on different parts of the body, palms, and soles (see Figure 17).

2.3. Diseases Causing Histopathological Changes in the Junction of Epidermis and Dermis. These diseases are focused mainly on ultrasonic sensors, which detect the base of papillary lines on the border of epidermis and dermis. The diagnoses also belong to the first group.

Hand eczema-particularly chronic forms (see above).

\section{Concrete Cases of Skin Diseases on Fingerprints}

Warts (verruca vulgaris) [10] are extremely common benign epidermal neoplasms that are caused by human papilloma viruses (HPVs). Warts commonly appear at sites of trauma, on the hand, in periungual regions. HPVs induce hyperplasia and hyperkeratosis. Large widespread warts occur in immunodeficient patients as well in patients with atopic eczema. The

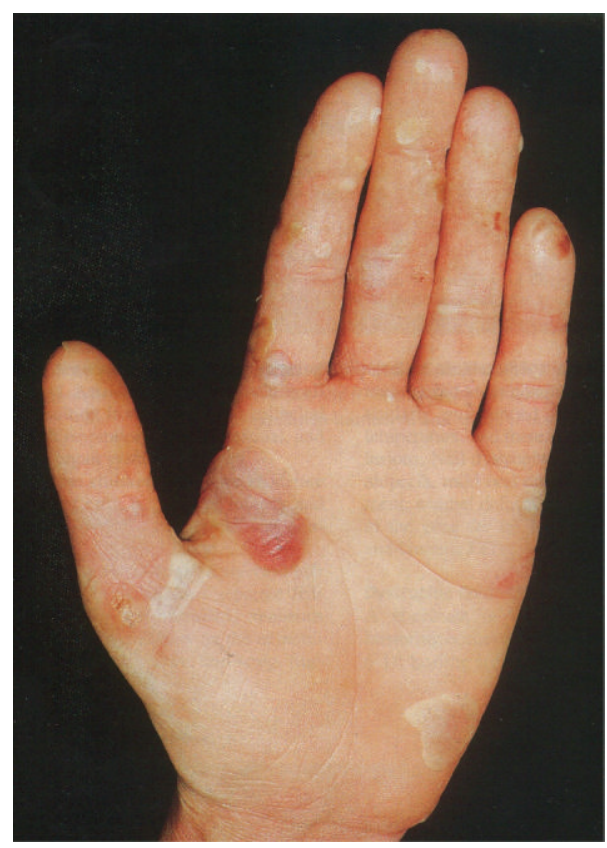

Figure 20: Epidermolysis bullosa [9, page 93].

aggressive surgical therapy may result in scarring. The lesions can affect all fingers of both hands; see Figure 18.

Psoriasis [9, 10, 12] (see Figure 19) is characterized by silvery-white scaly papules and plaques, sharply demarcated. It occurs in $1 \%$ to $3 \%$ of the population. The disease is transmitted genetically, and environmental factors are sometimes needed to precipitate the disease. The disease is lifelong and characterized by chronic, recurrent exacerbations and remissions that are emotionally and physically debilitating. Psoriasis of the palms and fingertips is characterized by red plaques with thick brown scale and may be indistinguishable from chronic eczema or tinea. The lamellar scales are more adherent than those on other parts of the body, and only their removal will reveal the reddish inflammatory base. There may be cracking and painful fissures and bleeding.

Systemic lupus erythematosus (SLE) [5] is a multisystem disease of unknown origin characterized by production of numerous diversity antibodies that cause several combinations of clinical signs, symptoms and laboratory abnormalities. The prevalence of LE in North America and Northern Europe is about 40 per 100,000 population. In the case of acute cutaneous LE, indurated erythematous lesions may be presented on palms.

Epidermolysis bullosa $[9,12]$ is a term given to groups of genetic diseases in which minor trauma causes noninflammatory blistering (mechanobullous diseases). Repetitive trauma may lead to a mitten-like deformity with digits encased in an epidermal "cocoon." These diseases are classified as scarring and nonscarring and histologically by the level of blister formation. Approximately 50 epidermolysis cases occur per million live births in the United States. The estimated prevalence in the United States is 8.2 per million, but this figure represents only the most severe cases as it does not 
include the majority of very mild disease going unreported (see Figure 20).

We have acquired fingerprints from patients suffering from different skin diseases. For this research it is also necessary to analyze a skin disease influence on specific fingerprint acquirement method, so all available methods for fingerprint obtaining were used: classic forensic fingerprinting method using dactyloscopic ink and card, clean fingerprinting using a chemical way for fingerprint acquirement on a special dactyloscopic paper, and set of electronic fingerprint sensors, each based on different scanning technology (optical, capacitive touch, and capacitive sweep technology).

Particular examples of selected skin diseases from our collection are shown in Figures 21, 22, 23, and 24. It is a good illustration for the difficulty, which is caused by skin disease for a fingerprint recognition system. It is nearly impossible to detect any papillary line (ridge) in the images (not even after use of image enhancement algorithms, e.g., [13]); therefore, the image enhancement algorithm in fingerprint recognition system is unable to reconstruct the papillary lines structures, and the image could not be processed further any more. The quality of such image is very low-due to different methodologies for estimation of the fingerprint quality, the image will be rejected at the beginning of the process. Indeed, this situation is very bad for the users- they cannot use the biometric systems; their physical identity has to be checked in another way.

The joint question to this topic is fingerprint image compression-the common compression methods for fingerprint images use JPEG-2000 or WSQ (wavelet scalar quantization), whereas other possible compression scenarios exist [14]. It is unclear how the compression algorithm can influence the quality of such fingerprint image, containing a skin disease.

The most common skin diseases, which we collected, are, psoriasis, atopic eczema, verruca vulgaris, and pulpitis sicca. The probability of occurrence of other skin diseases is lower. However, the quality of other skin diseases, which we collected, was comparable with the types shown in Figures 21, 22,23 , and 24 . In the most cases, nearly no papillary lines (ridges) are identifiable; that is, such fingerprints are not suitable for further processing (the pattern recognition algorithm, e.g., [15], is unable to detect biometric patterns necessary for further processing and recognition).

\section{Fingerprint Image Enhancement Algorithm}

Based on the experience with the damaged fingerprints presented in the previous chapter, we tried to propose an algorithm, which could be used for enhancement of fingerprint images distorted by a skin disease. The process of the fingerprint (with a skin disease) image enhancement is shown in Figure 25.

The first step is to remove the background information, which does not belong to the fingerprint itself and to estimate the image quality. This is not a difficult task, because we do not use dactyloscopic cards, in which much background information/noise could be found. For the estimation of the
TABLE 1: The acquired database NFIQ rate testing results.

\begin{tabular}{lccc}
\hline Disease/NFIQ & Min. & Max. & Avg. \\
\hline Verruca vulgaris & 1 & 5 & 2,93 \\
Atopic dermatitis & 1 & 5 & 3,61 \\
Psoriasis & 3 & 5 & 4,92 \\
\hline
\end{tabular}

quality, we use our own methodology [16], which is based on the thickness of the ridges and valleys (papillary lines) and the steepness of the upward and downward edges of the papillary lines.

One of our own criteria for the image quality estimation is similarity with the sine function (this is only one possibility from a set of other possible functions) [16]:

$$
D_{D}=\left(\frac{A_{\mathrm{FP}}}{A_{\mathrm{sin}}}-1\right) \cdot 100 \%,
$$

where

$$
\begin{aligned}
A_{\mathrm{FP}} & =\int_{x_{S}}^{x_{E}} f(x) d x, \\
A_{\text {sin }} & =\int_{x_{S}}^{x_{E}} \sin (x) d x,
\end{aligned}
$$

whereas the function $f(x)$ represents a side cut of a papillary line-relation between the position and intensity of the pixel.

The second criterion is the thickness of the papillary line, defined as follows [16]:

$$
D_{\mathrm{Th}}=\left(\frac{\mathrm{Th}}{0.033}-1\right) \cdot 100 \% \text {, }
$$

with

$$
\mathrm{Th}=\frac{2.54}{R_{\mathrm{DPI}}} \cdot N_{\mathrm{Pix}}[\mathrm{cm}],
$$

whereas $R_{\mathrm{DPI}}$ is the resolution of the sensor and $N_{\text {Pix }}$ is number of pixels of the papillary line in a defined span. The average thickness of the papillary line has been experimentally established to be $0.33 \mathrm{~mm}$.

The last criterion is the steepness of the papillary line. Here we define only the steepness of the upward papillary line (the deduction for downward line is simple) as follows [16]:

$$
D_{\alpha}=\frac{\left|\alpha-60^{\circ}\right|}{60^{\circ}} \cdot 100 \%
$$

with

$$
\alpha=\arcsin \left(\frac{P_{x_{1}}}{\sqrt{P_{x_{1}}+P_{y}}}\right),
$$

where the values $P_{\text {? }}$ are defined according to Figure 26 .

Furthermore, we use NFIQ (NIST fingerprint image quality) and other standardized methods for fingerprint image quality estimation. We already have the NFIQ test results. Some basic statistical results of the NFIQ rate of acquired 

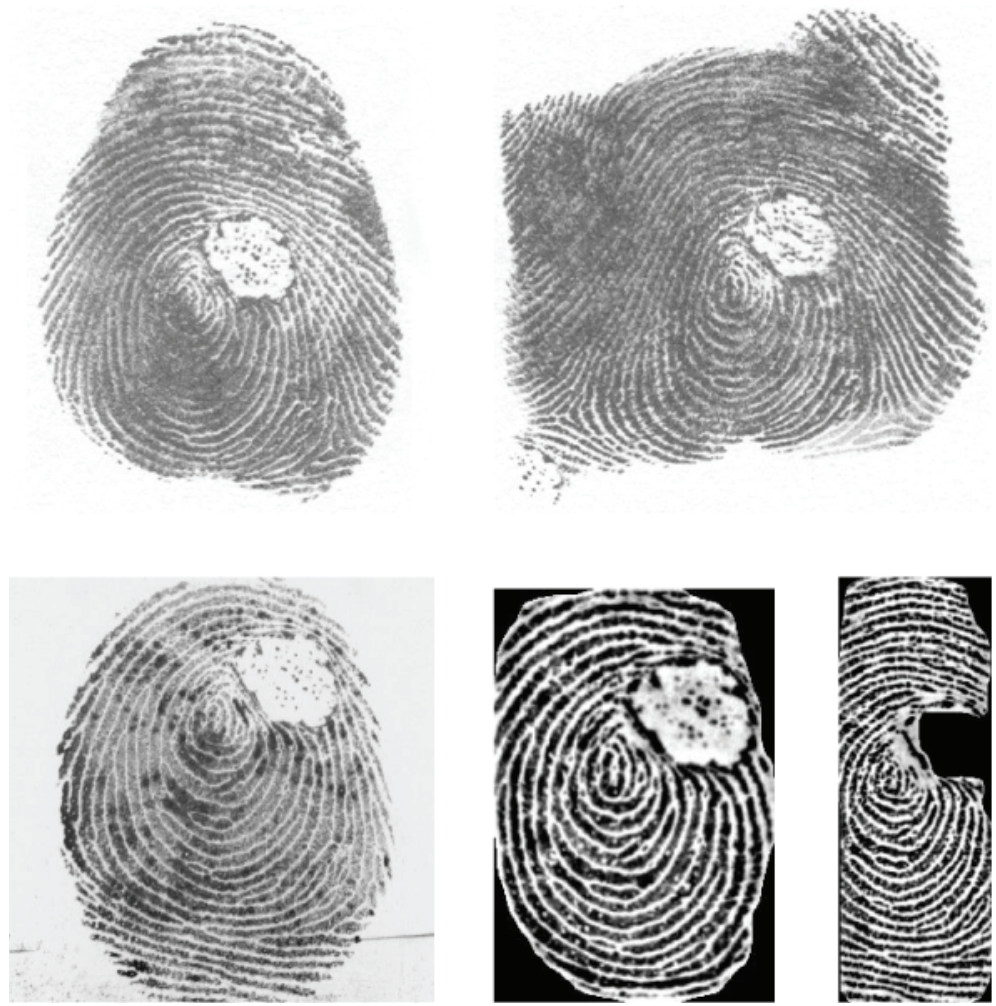

FIGURE 21: Fingerprints with warts—verruca vulgaris (same person).
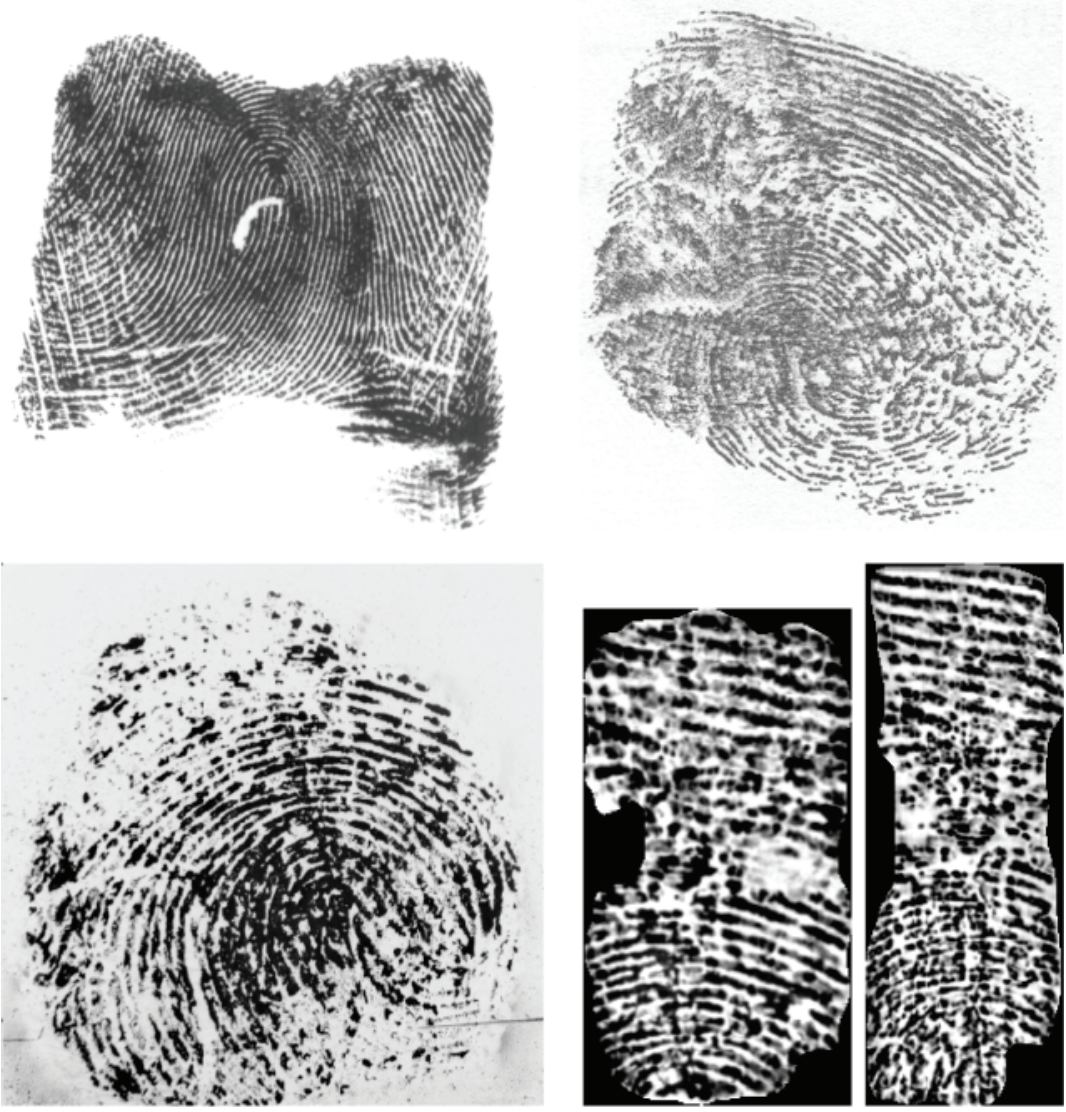

FIGURE 22: Fingerprints with atopic eczema (different people). 

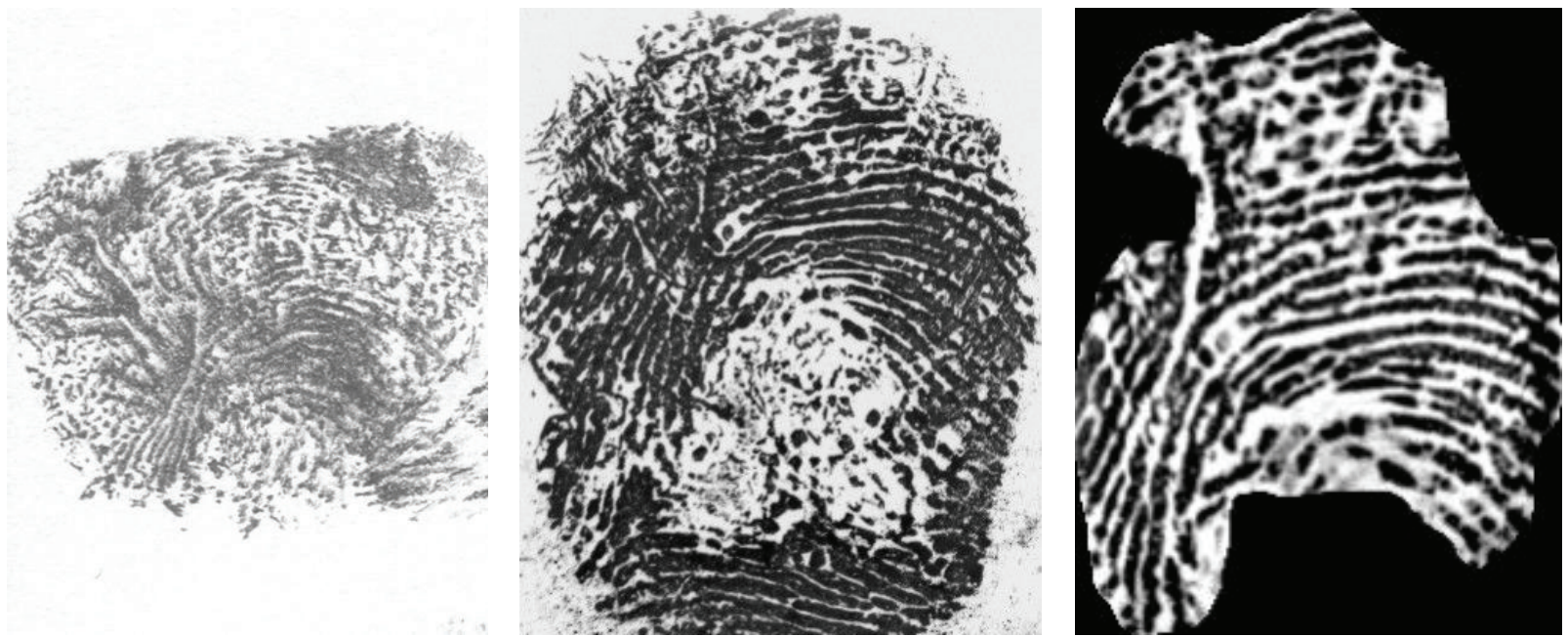

FIGURE 23: Fingerprints with light psoriasis (same person).
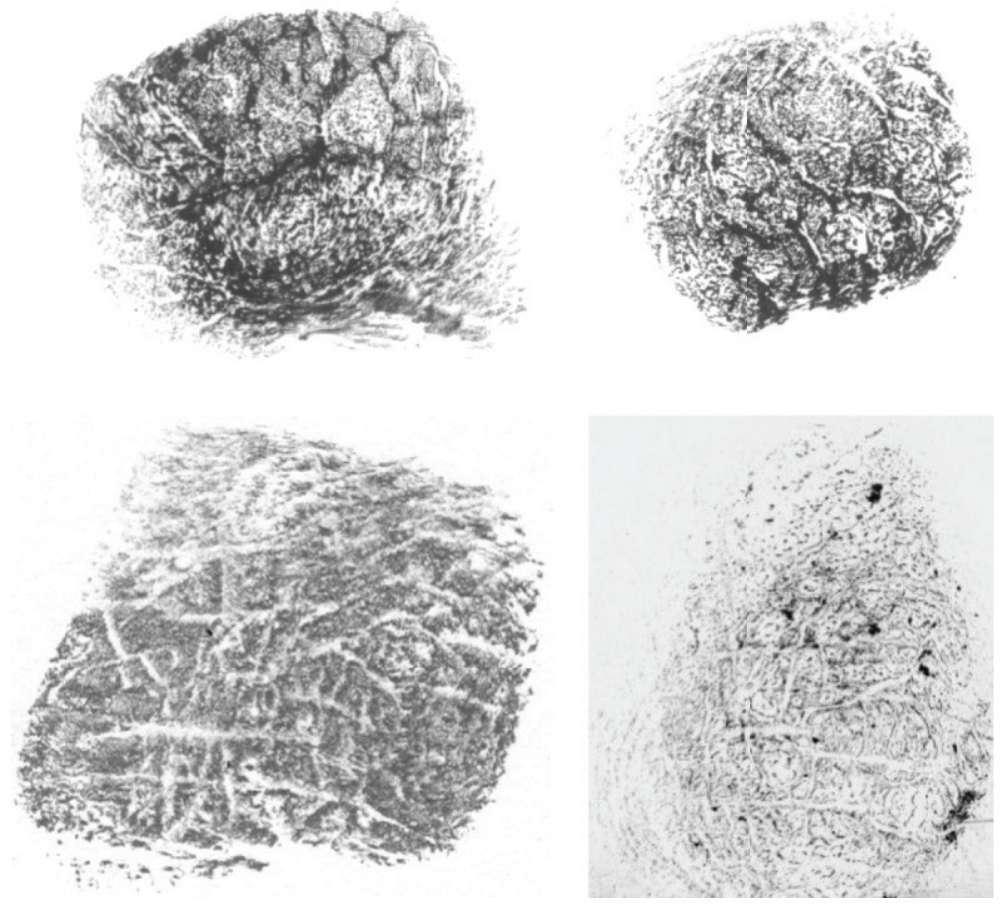

FIGURE 24: Fingerprints with advanced psoriasis (different people).

database are shown in Table 1. NFIQ rate marks the top quality fingerprints with number 1 and the worst quality fingerprints with number 5 . The performed testing confirmed that the amount of damages on the finger corresponds to the NFIQ rate, because the least invasive diseases (verruca vulgaris) have statistically better results than the serious invasive diseases like psoriasis. All other methods are being tested at the moment; that is, we are trying to find such solution, which would be reliable and quick enough to be usable in a stand-alone device operated by a microcontroller unit with a relatively low processing power.
There are three possible results given by the image quality estimator-LOW, MIDDLE, HIGH. If the quality is $H I G H$, then there is no need for any complex image quality enhancement; that is, in this case the image could be directly processed by a minutia extraction algorithm. If the quality is $L O W$, the image contains irrecoverable fragments in the fingerprint; that is, the enhancement is unreasonable in this case. Our enhancement algorithm is needed for the images classified as MIDDLE quality images, of which the quality is insufficient for a direct processing and is not that bad not to be possible to enhance. 


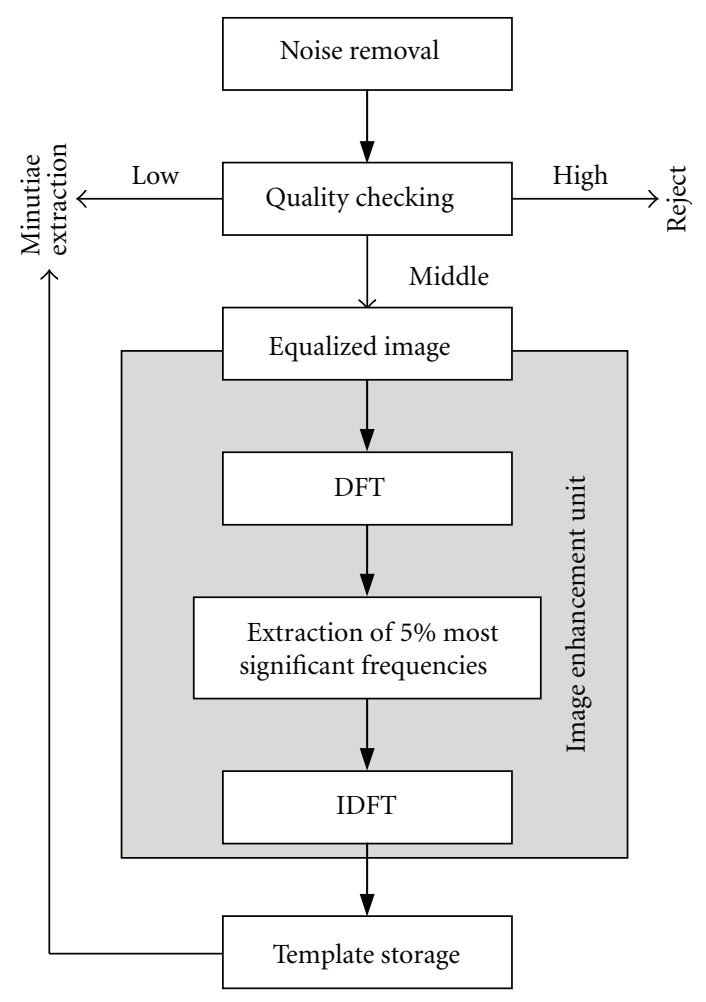

Figure 25: Process of the fingerprint image enhancement.

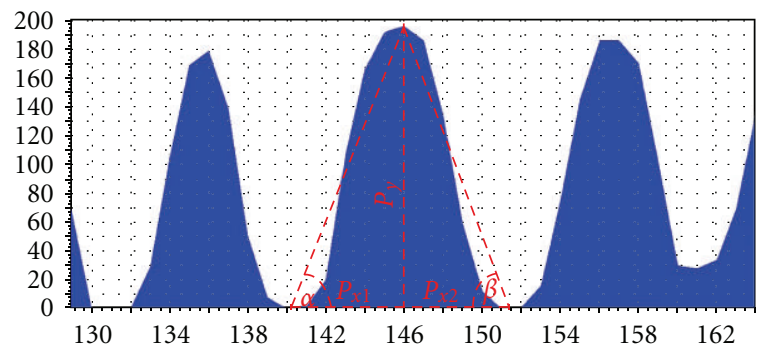

Figure 26: Determination of the upward and downward sections of the curve in a side cut of a papillary line provided by the fingerprint image.

The input image of our image quality enhancement algorithm should be an equalized image. The extractor finds the first stable point in the image. Then it cuts a subarea of an image undersampled to $100 \times 100$ pixels. This subarea is processed by a local equalization algorithm (see Figure 27). Then, a phase image is obtained by the discrete fourier transform (DFT). In our case, only $5 \%$ of the most significant frequencies (see Figure 2(b)) are being taken into account for the further processing. We use these frequencies as an add-on for the later comparison as well.

Our extractor undersamples the fingerprint image, and then it extracts the background of the image-there are valleys for standard fingerprints, scars, wrinkles, and other damages for critical fingerprints. Only the smaller part of the image (around expected core position) is used for creation of the phase images.

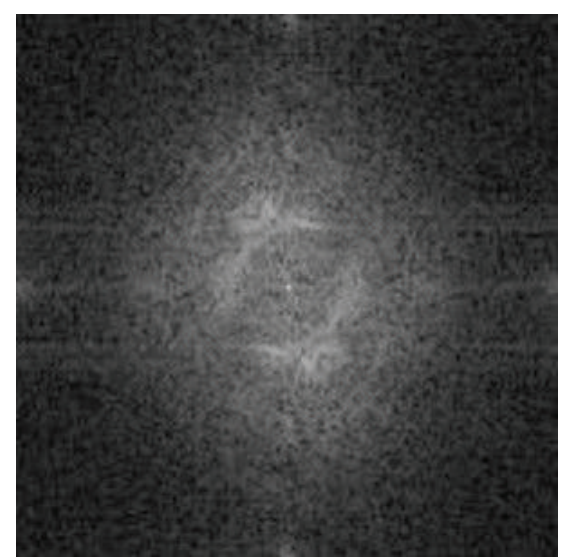

(a)

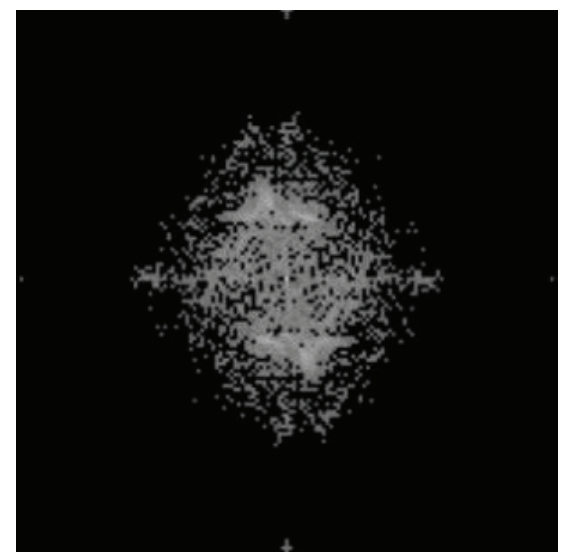

(b)

Figure 27: Phase image (a) and phase image with most significant frequencies (b).

Each feature extractor outputs a vector of numbers. To measure similarity of the vectors, the Euclidian distance and square error between two vectors are calculated. For the contour shape, a special comparator was designed, which puts greater weights to the tangents that are near the fingertip. They characterize more the uniqueness of the contour shape, in our opinion.

Hence, the template is represented by $5 \%$ of the most significant frequencies. The feature image of current record is represented by all of frequencies (100\%). Now we calculate the phase correlation of these two images:

$$
C_{\text {phase }}=\frac{\mathrm{DFT}_{\text {image }} \cdot \mathrm{DFT}_{\text {template }}^{*}}{\left|\mathrm{DFT}_{\text {image }} \cdot \mathrm{DFT}_{\text {template }}^{*}\right|},
$$

where DFT is the discrete Fourier transform of the respective image and DFT* is its complex conjugate. All multiplications in the equation are simple per element operations, which can be finished really quickly.

After the phase correlation of the template image and the current image, the $C_{\text {phase }}$ is transformed by applying the inverse DFT to get the normalized cross-correlation $C_{\text {color }}$ of both of the images (see Figure 28). The image $C_{\text {color }}$ is then normalized again to transform the values to an interval from 


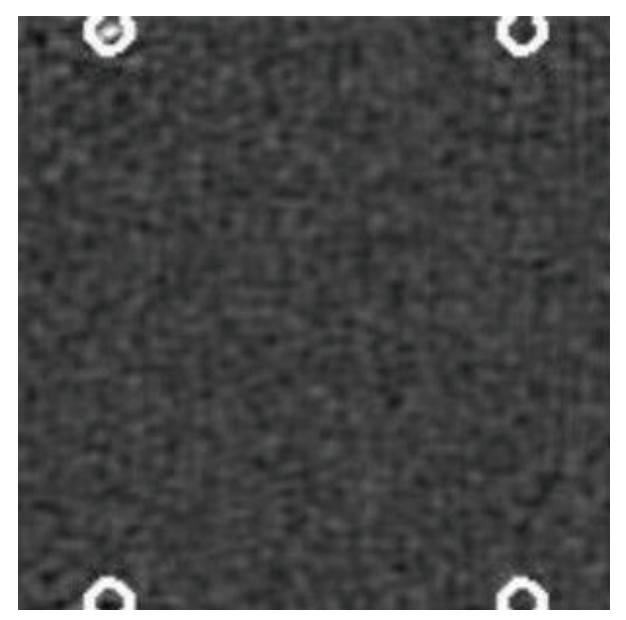

FIGURE 28: Normalized cross-correlation image in the color space with a marked global peak in it (upper left corner).

0 to 1 . The goal is to determine the location of the global peak in the $C_{\text {color }}$ image. Based on the value of the peak and according to the mean value, the final score is obtained as

$$
\text { score }=\frac{1}{\text { Peak }- \text { Mean }}-1 .
$$

The best final score is 0; the Mean value is computed from the selected pixel intensities. The selected pixels have their intensities from $2 \%$ to $98 \%$. In that case the highest and the lowest peak intensities are avoided for the computation of the Mean value. Therefore, $C_{\text {color }}$ images with obvious peaks generate scores that are better separated from those with lower peaks or without peaks at all. In other words, the genuine images are better separated from the impostor images.

\section{Conclusion}

It is clear that either the color of the skin or the structure of papillary lines on the fingertip could be influenced by the skin diseases. If only the color has changed, some of optical fingerprint scanners might be influenced, and so this change is not crucial. On the other hand, the change of skin structure is very significant, because, if papillary lines are damaged, it is impossible to find the minutiae and therefore to recognize the person. If we are unable to recognize/enroll a person, then such person cannot use the biometric system based on the fingerprint recognition technology, and therefore there is a big problem security issue- - how to authorize such person, if use of PINs (personal identification numbers) or other authorization methods is out of question? We do not consider other security issues, like secure communication [17], revocable biometrics, template protection, and so forth-these are out of scope of this paper.

Some of these diseases are only temporary; that is, after the healing of such disease, the papillary line structure or color is restored and the user is again able to use their fingers for the fingerprint recognition for the authorization tasks in security systems. However, some diseases leave irrecoverable finger damage restraining new growth of papillary lines, and respective user is then unable to use their fingerprints for appropriate recognition tasks in automated fingerprint security systems.

We prepare a new collection of fingerprints with skin diseases from patients, oriented not only on dactyloscopic fingerprints but also on live fingerprint images from different fingerprint scanners. The resulting images will be used for quality assessment and, if the quality will be acceptable, for minutiae extraction and comparison based on minutiae. Maybe, we will consider other methods, based not only on minutiae but on correlation or other methods as well.

\section{Acknowledgments}

This research has been realized under the support of the following grants: "Security-Oriented Research in Information Technologys-" MSM0021630528 (CZ), "Information Technology in Biomedical Engineering-" GD102/09/H083 (CZ) and "Advanced secured, reliable and adaptive IT-" FIT-S-11-1 and "The IT4Innovations Centre of Excellence-" IT4I-CZ 1.05/1.1.00/02.0070.

\section{References}

[1] "Evaluation of Fingerprint Recognition Technologies - BioFinger," Public Final Report, version 1.1, Bundesamt für Sicherheit in der Informationstechnik, p. 122, 2004.

[2] A. K. Jain, P. Flynn, and A. A. Ross, Handbook of Biometrics, Springer, New York, NY, USA, 2008.

[3] R. M. Bolle, J. H. Connell, S. Pankanti, N. K. Ratha, and A. W. Senior, Guide to Biometrics, Springer, New York, NY, USA, 2004.

[4] W. D. James, T. G. Berger, and D. M. Elston, Andrew's Diseases of the Skin-Clinical Dermatology, Elsevier Saunders, Ontario, Canada, 10th edition, 2006.

[5] T. P. Habif, Clinical Dermatology, Mosby, Hong Kong, China, 4th edition, 2004.

[6] "The Science of the Skin," http://www.naturalrussia.com/natural/skin/structure.html.

[7] M. Drahanský, E. Březinová, D. Lodrová, and F. Orság, "Fingerprint recognition influenced by skin diseases," International Journal of Bio-Science and Bio-Technology, vol. 3, no. 4, pp. 1122, 2010.

[8] E. Březinová, M. Drahanský, and F. Orság, "Dermatologic diseases and fingerprint recognition," in Database Theory and Application, Bio-Science and Bio-Technology 2010, pp. 251-257, Springer, Berlin, Germnay, 2010.

[9] K. Wolff, R. A. Johnson, and D. Suurmond, Fitzpatrick's Color Atlas and Synopsis of Clinical Dermatology, McGraw-Hill, New York, NY, USA, 5th edition, 2005.

[10] J. Štork et al., Dermatovenerologie, Galén, Prague, Czech Republic, 2008.

[11] O. Braun-Falco, G. Plewig, H. H. Wolff, and W. H. C. Burgdorf, Eds., Dermatology, Springer, Heidelberg, Germany, 2nd edition, 2000.

[12] W. L. Weston, A. T. Lane, and J. G. Morelli,, Color Textbook of Pediatric Dermatology, Mosby Elsevier, Hong Kong, China, 2007.

[13] I. Jafar and H. Ying, "New algorithms for contrast enhancement in grayscale images based on the variational definition 
of histogram equalization," Integrated Computer-Aided Engineering, vol. 15, no. 2, pp. 131-147, 2008.

[14] A. A. Mohammed, R. Minhas, Q. M. J. Wu, and M. A. SidAhmed, "An efficient fingerprint image compression technique based on wave atoms decomposition and multistage vector quantization," Integrated Computer-Aided Engineering, vol. 17, no. 1, pp. 29-40, 2010.

[15] H. Lee, E. Kim, and M. Park, "A genetic feature weighting scheme for pattern recognition," Integrated Computer-Aided Engineering, vol. 14, no. 2, pp. 161-171, 2007.

[16] M. Drahanský, Fingerprint recognition technology: liveness detection, image quality and skin diseases, Habilitation thesis, Brno, Czech Republic, 2010.

[17] D. Neumann, R. Eckmiller, and O. Baruth, "Combination of biometric data and learning algorithms for both generation and application of a secure communication link," Integrated Computer-Aided Engineering, vol. 14, no. 4, pp. 345-353, 2007. 

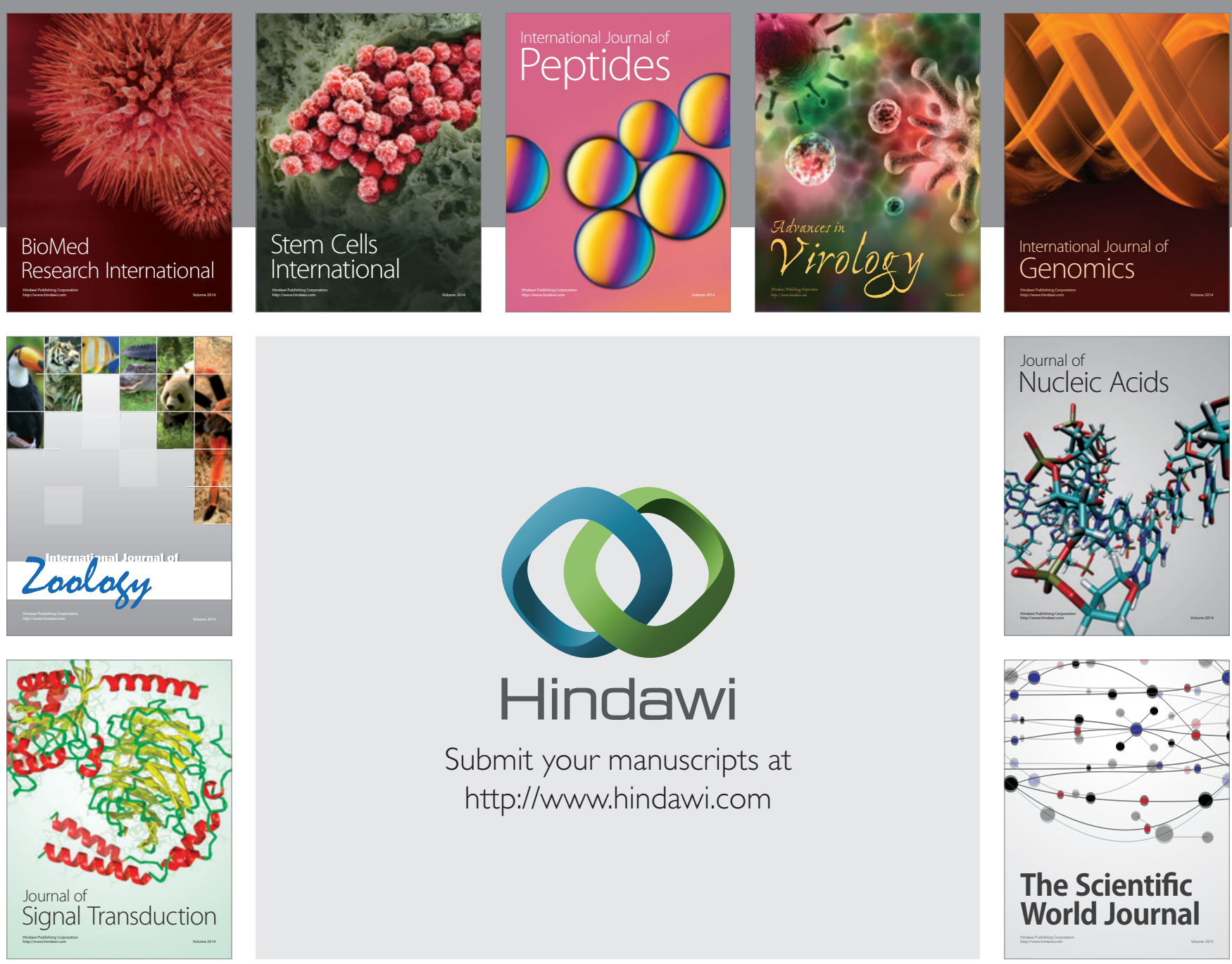

Submit your manuscripts at

http://www.hindawi.com
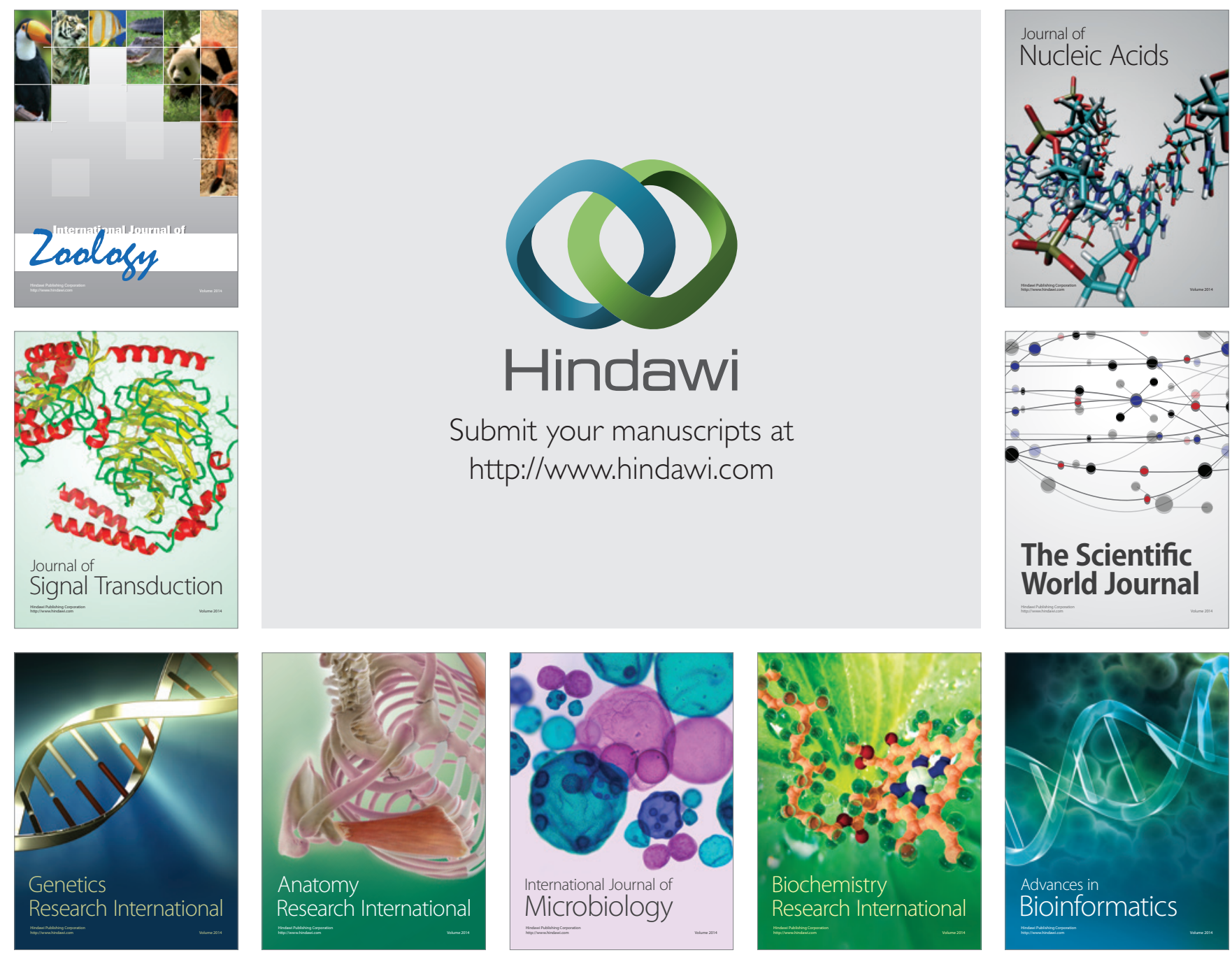

The Scientific World Journal
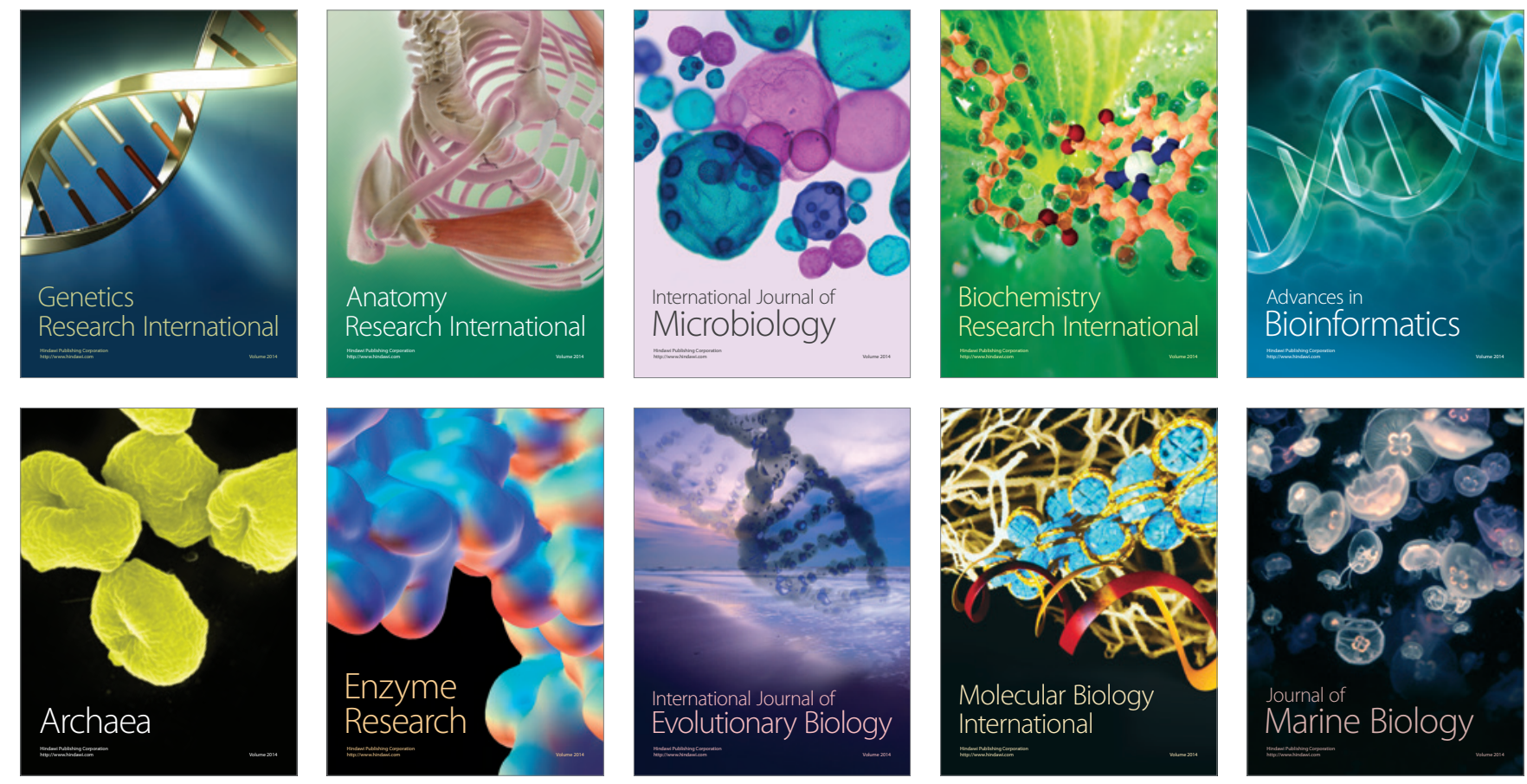